\title{
Observation of coherent zone-folded acoustic phonons generated by Raman scattering in a superlattice
}

\author{
P. Hawker, ${ }^{\text {a) }}$ A. J. Kent, and L. J. Challis \\ School of Physics and Astronomy, University of Nottingham, University Park, Nottingham, \\ NG7 2RD United Kingdom \\ A. Bartels, T. Dekorsy, and H. Kurz \\ Institut für Halbleitertechnik, Sommerfeldstr. 24, 52074 Aachen, Germany \\ K. Köhler \\ Fraunhofer-Institut für angewandte Festkörperphysik, D-79108 Freiburg, Germany
}

\begin{abstract}
We have used pulse time-of-flight techniques to examine the phonon emission from an optically excited GaAs/AlAs superlattice structure. For laser excitation wavelengths shorter than $767 \mathrm{~nm}$ (the energy of $E 1 H H 1$ transition), we detect a significant longitudinal acoustic phonon component directed in a narrow beam normal to the structure. Under identical excitation conditions, generation of coherent longitudinal acoustic phonons has previously been observed in this structure. We suggest that the excitation wavelength and angular characteristics of the longitudinal acoustic emission is consistent with those of propagating modes produced as coherent phonons "leak" from the superlattice structure.
\end{abstract}

In recent years a number of experiments have been reported in which coherent phonons have been generated in semiconductor structures using ultrafast laser pulses. In the first experiments ${ }^{1}$ coherent optical phonons were generated. However, more recently, coherent tetrahertz acoustic phonons have been generated by ultrafast excitation of electrons and holes in GaAs/AlGaAs quantum wells ${ }^{2}$ and superlattices ${ }^{3,4}$ and detected by surface deflection ${ }^{2}$ and timeresolved reflectivity. ${ }^{3,4}$

Specifically, it has been shown that coherent zone-folded longitudinal acoustic (LA) phonons are excited when a femtosecond laser pulse is shined onto a superlattice., ${ }^{3,4}$ The excitation process is attributed to impulsively stimulated Raman scattering which is expected to exhibit a strong resonant enhancement where the formation of an electron-hole pair is accompanied by the creation of a LA phonon. The acoustic miniband structure of the superlattice permits the creation of relatively high frequency phonons of low $q$ with the principal feature being a triplet of phonon modes centered around $q$ $\sim 0$ with sidebands at $q \sim 2 k_{\text {laser }}$ produced by backscattering. The central frequency corresponds to the unfolded zone boundary at $q=2 \pi / d_{\mathrm{SL}}$, where $d_{\mathrm{SL}}$ is the period of the superlattice, leading to $\omega \approx 2 \pi c_{\mathrm{LA}} / d_{\mathrm{SL}}$, where $c_{\mathrm{LA}}$ is the velocity of the LA phonons and, hence, $\nu=c_{\mathrm{LA}} / d_{\mathrm{SL}}$ which is typically around $500 \mathrm{GHz}$ for the structures used. A triplet of modes centered on twice this frequency corresponding to the unfolded zone boundary at $q=4 \pi / d_{\mathrm{SL}}$ is also observed with an intensity more than ten times weaker.

The presence of the phonons has been observed by studying the reflection of a probe pulse from the sample surface following the excitation pulse. ${ }^{3,4}$ The reflected signal amplitude oscillates at a few hundred gigahertz which is attributed to modulation of the refractive index by the lattice

${ }^{a)}$ Electronic mail: Philip.Hawker@nottingham.ac.uk vibrations. The modulation amplitude decays in a few nanoseconds. This is likely to be the result of the confined modes leaking into propagating monochromatic phonons. This has been observed in optical two-color pump-probe experiments but only over small distances $(500 \mathrm{~nm})^{5}$ and not in phonon spectroscopy using incoherent detection schemes. In this work our aim has been to observe these propagating phonons directly using pulsed time-of-flight techniques.

The sample was fabricated from the same wafer material as used for sample A in Refs. 3 and 4, namely, a 40 period GaAs/AlAs superlattice (each period $22 \mathrm{ML}$ of GaAs with 4 ML of AlAs) grown by molecular beam epitaxy on a $500 \mu \mathrm{m}$ (100) semi-insulating GaAs substrate. On the opposite surface to the superlattice was fabricated a $10 \times 10 \mu \mathrm{m}$ thin film superconducting aluminum bolometer to detect the phonons. With the sample held in a liquid helium cryostat at the bolometer transition temperature $(\sim 2 \mathrm{~K})$ the superlattice was excited with femtosecond pulses from a tunable Ti:sapphire laser, Fig. 1. Emitted phonons then propagated across the substrate to the bolometer raising its temperature slightly and causing a small change in resistance. With a small bias current flowing this gave a transient voltage signal that could be averaged to produce a phonon time-of-flight signal, Fig. 2 . By moving the laser excitation spot over the surface it was possible to investigate the angular distribution of the emitted phonons.

For reasonable temporal resolution in the time-of-flight measurements the time during which excitation occurs must be small in comparison with the phonon flight time to the bolometer. The Ti:sapphire laser pulse repetition rate was fixed at $80 \mathrm{MHz}(\sim 12 \mathrm{~ns}$ between pulses), or essentially continuous on the time scale of phonon flight time $(\sim 100$ ns). It was necessary therefore to use an external acousticoptic switch. With this it was possible to isolate individual pulses from the laser. 


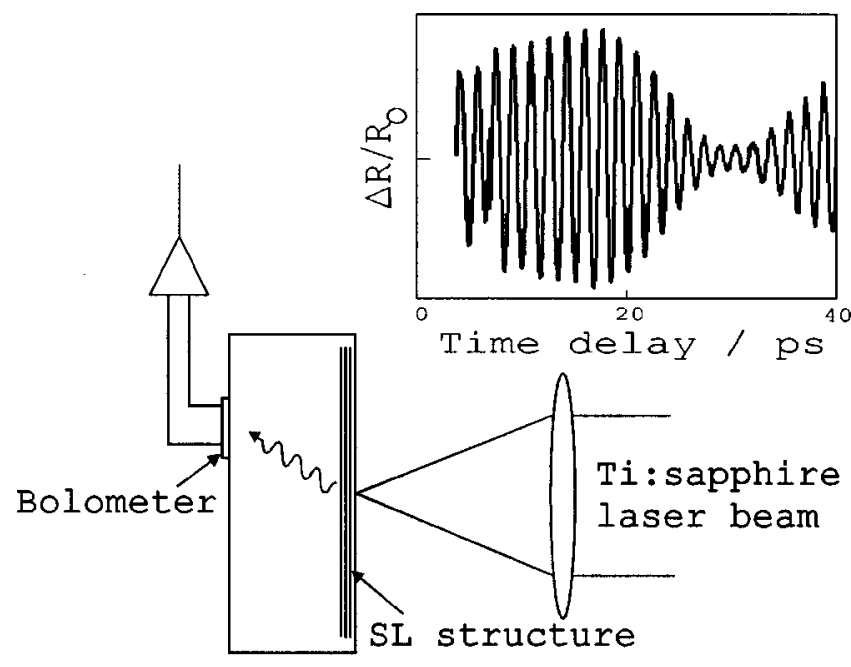

FIG. 1. Experimental setup. Inset: Oscillatory changes in the surface reflectivity measured by the technique described in [A. Bartels et al., Phys. Rev. Lett. 82, 1044 (1999)] and attributed to the presence of coherently generated LA phonons. The period gives the coherent phonon frequency. The signal exhibits strong beating between several excited coherent modes.

Figure 2 shows a number of phonon time-of-flight traces taken at various excitation wavelengths with the laser spot directly opposite the bolometer. We interpret such traces as follows. At time zero the excitation impinges upon the sample producing electrons and holes in the superlattice and surrounding GaAs substrate/capping layer material. A large peak is observed coincident with the laser pulse which changes little with laser wavelength, and which is at a maximum when the laser excitation point is directly opposite the bolometer. We attribute this to excitation of subband gap luminescence which propagates through the substrate unattenuated and is detected by the bolometer. Relaxation of the free carrier density occurs with a time constant of some 100 ps accompanied by the emission of incoherent phonons, normally observed as carriers relax to the band edges, and the generation of coherent LA modes. A short time later emitted phonons arrive at the bolometer and are detected. The pre-

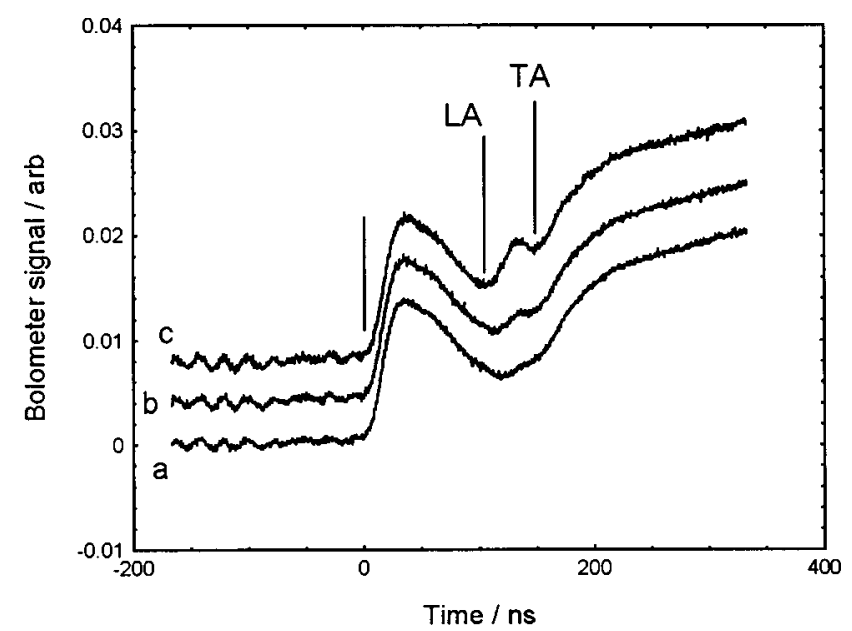

FIG. 2. Typical phonon time-of-flight traces taken at laser wavelengths of (a) $785 \mathrm{~nm}$ (excitation energy below the superlattice ground state resonance), (b) $767 \mathrm{~nm}$ (on resonance), and (c) $755 \mathrm{~nm}$ (energy above resonance). The arrival times of the ballistic LA and TA phonons are indicated. These mark the start of the rise of the signals.

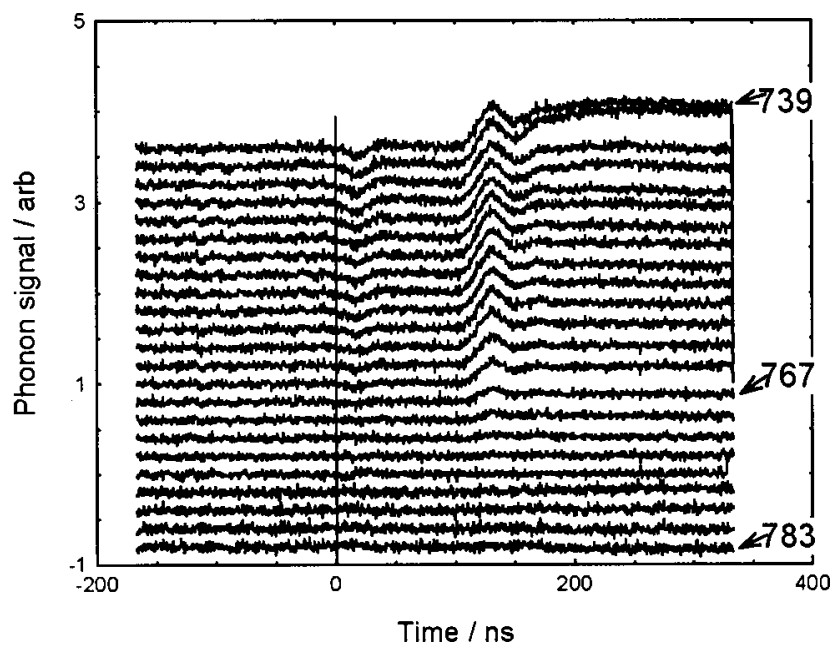

FIG. 3. A series of time-of-flight traces taken in $2 \mathrm{~nm}$ steps over the range $783-739 \mathrm{~nm}$. The LA signal appears as carriers become excited in the superlattice.

dicted ballistic LA and transverse acoustic (TA) phonon arrival times are marked.

In Refs. 3 and 4 excitation of coherent LA phonons with $450 \mathrm{GHz}$ frequency was observed with the laser tuned to the first superlattice interband transition $(E 1 H H 1)$ corresponding to an excitation wavelength of $767 \mathrm{~nm}$. (The band gap of GaAs at liquid helium temperatures corresponds to an excitation wavelength of $816 \mathrm{~nm}$.) For excitation wavelengths, $\lambda$ longer than $767 \mathrm{~nm}$ (excitation in the GaAs substrate only) we observe negligible LA emission but a strong TA mode signal with a long tail, Fig. 2. The signal is consistent with previous measurements ${ }^{6}$ in bulk GaAs and is indicative of strong longitudinal optical (LO) phonon emission in the substrate. Highly excited carriers relax by emitting a cascade of LO phonons before reaching the GaAs band edge, recombining with holes and emitting a band gap photon. The LO phonons decay rapidly via a series of steps to high frequency TA modes $(\nu \sim 1.5 \mathrm{THz})$. Strong isotope scattering within the substrate then gives rise to some diffusive propagation and the observed long time tail to the signal.

For excitation at wavelengths below $767 \mathrm{~nm}$ (carriers now excited in the superlattice as well as the substrate), we observe in addition, the appearance of a sharp LA mode peak. Using a trace taken at a wavelength much longer than $767 \mathrm{~nm}$ as a reference we can subtract off the direct optical excitation peak to reveal the LA mode more clearly, Fig. 3. The rapid turn-on of the LA mode emission as carriers become excited in the superlattice is shown more vividly in Fig. 4, where we plot the integrated LA mode intensity versus $\lambda$. Negligible LA emission occurs for $\lambda>767 \mathrm{~nm}$. LA emission increases rapidly as carriers become excited in the superlattice $(\lambda=767 \mathrm{~nm})$ and then the emission intensity remains approximately constant as $\lambda$ decreases further. The weak structure present in the region $\lambda<767 \mathrm{~nm}$ was reproducible and we note that the peak at $\lambda=750 \mathrm{~nm}$ is separated from the ground state superlattice resonance $(E 1 H H 1)$ by $36 \mathrm{meV}$, or the energy of a bulk GaAs LO phonon. We have no definitive explanation but suggest that the feature may be associated with a resonant absorption process; carrier excitation in the well accompanied by emission of a GaAs LO phonon. Such a process would increase the capture cross 


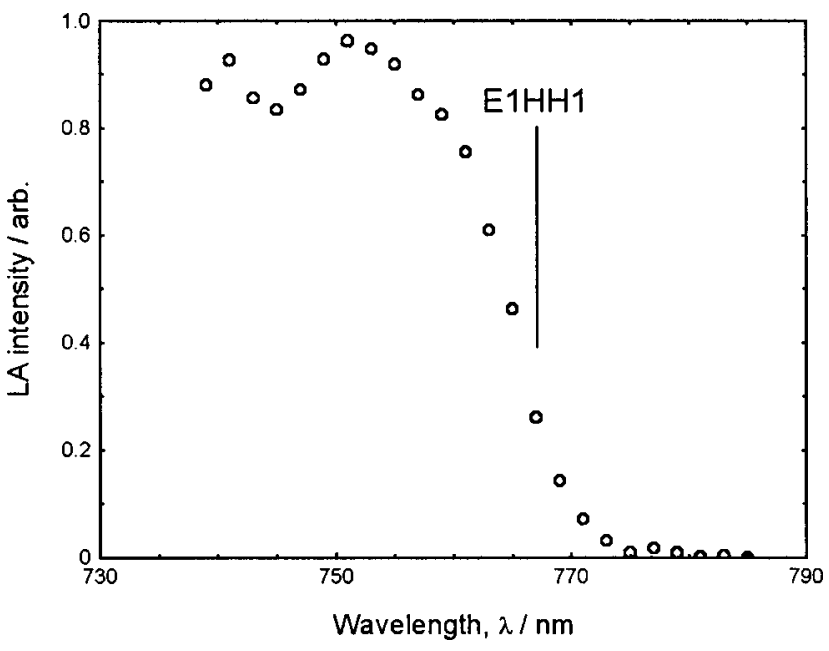

FIG. 4. Integraed LA mode intensity plotted against excitation wavelength.

section for carrier generation resulting in an increased detected signal.

Figure 5 shows the angular dependence of the LA mode signal at $\lambda=760 \mathrm{~nm}$. Bearing in mind that LA phonons are only weakly focused in GaAs, we note that the emission is strongly peaked normal to the plane of the superlattice. This observation is inconsistent with previous studies of phonon emission from hot carriers confined in a quantum well $(\mathrm{QW}){ }^{7}$ In that case, screening and momentum conservation

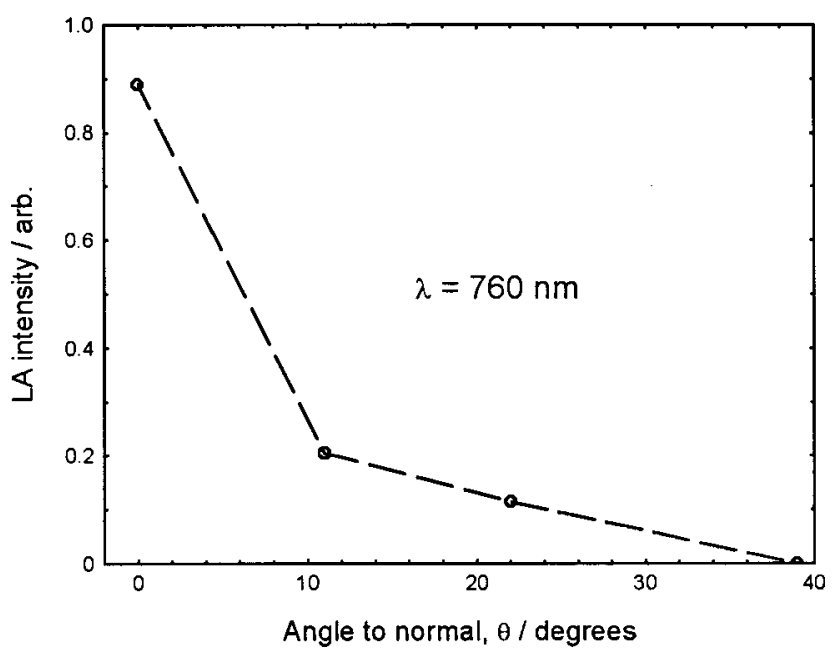

FIG. 5. Angular dependence of the LA signal. results in a negligible LA intensity emitted normal to the two dimensional electron gas, the emission instead being concentrated in a cone around the normal direction. The cone half angle, $\theta$, is related strongly to the $\mathrm{QW}$ width, $d$. In our samples we estimate the induced sheet carrier density to be comparable with that assumed in Ref. 7. Then, taking $d \sim 5$ $\mathrm{nm}$, gives a predicted peak in emission intensity at $\theta \sim 30^{\circ} .7$ No such feature is evident in our data. Instead, we suggest that the observed distribution of LA emission is consistent with that from a coherent source with a lateral extent large on the scale of the phonon wavelength $(\sim 10 \mathrm{~nm}$ at 450 $\mathrm{GHz}$ ). For such a source diffraction effects would be insignificant, and the phonons would be emitted in a narrow beam normal to the source plane. Assuming that in our case the source size equals the diameter of the focus laser spot $(\sim 50$ $\mu \mathrm{m})$ then a narrow beam of emission would be expected. We suggest therefore that the observed LA signal present for excitation wavelengths below $767 \mathrm{~nm}$ is due to the propagating component produced as coherently generated phonons "leak" from the superlattice structure.

Further experiments are underway to examine the LA emission in greater detail using a detector with spectral discrimination. Using such a detector it should be possible to unambiguously confirm that the frequency of the detected propagating phonons is the same as that of the coherent modes generated in the previous optical studies.

The authors would like to thank the CLRC Lasers for Science facility for the loan of the femtosecond Ti:sapphire laser used in this experiment. The authors would also like to thank the School of Physics and Astronomy, University of Nottingham for additional financial support and R. Bellingham for assistance in taking the data.

${ }^{1}$ W. E. Bron, J. Kuhl, and B. K. Rhee, Phys. Rev. B 34, 6961 (1986); G. C. Cho, W. Kütt, and H. Kurz, Phys. Rev. Lett. 65, 764 (1990); also see for an overview T. Dekorsy, G. C. Cho, and H. Kurz, in Light Scattering in Solids VIII, edited by M. Cardona and G. Güntherodt (Springer, Berlin, 2000), and references therein.

${ }^{2}$ J. J. Baumberg, D. A. Williams, and K. Kohler, Phys. Rev. Lett. 78, 3358 (1997).

${ }^{3}$ A. Yamamoto, T. Mishina, and Y. Masumoto, Phys. Rev. Lett. 73, 740 (1994).

${ }^{4}$ A. Bartels, T. Dekorsy, H. Kurz, and K. Kohler, Phys. Rev. Lett. 82, 1044 (1999).

${ }^{5}$ K. Mizoguchi, M. Hase, and S. Nakashima, Phys. Rev. B 60, 8262 (1999).

${ }^{6}$ R. G. Ulbrich, V. Narayanamurti, and M. A. Chin, Phys. Rev. Lett. 45, 1432 (1980)

${ }^{7}$ Cz. Jasiukiewicz, D. Lehmann, A. J. Kent, A. J. Cross, and P. Hawker, Physica B 263-264, 183 (1999). 